\title{
AFFINE CONORMAL OF CONVEX HYPERSURFACES
}

\author{
CHI-MING YAU \\ (Communicated by Jonathan M. Rosenberg)
}

\begin{abstract}
The geometry of convex hypersurfaces in real affine space is analyzed using the affine conormal. A weak version of Chern's conjecture, characterizing paraboloids among convex graphs, is proved. In addition, it is shown that a closed convex affine hypersurface with constant affine total curvature is an ellipsoid.
\end{abstract}

That the Gauss map is the central focus for the geometry of Euclidean submanifolds is well-known. We would like to find an analogue for the geometry of convex hypersurfaces under the group of unimodular affine transformation.

There are two immediate candidates: the affine normal and conormal. The latter is preferred as it has a simple Laplacian formulae, which yields a Bernstein Theorem (a weak version of Chern's Conjecture) almost immediately. Furthermore, the affine conormal defines a new convex hypersurface when the affine third fundamental form is positive definite. As an even closer analogue to the Euclidean case, the new hypersurface turns out to be an elliptic affine hypersphere iff the affine total curvature of the old one is constant. From this we get the

Theorem. A closed convex affine hypersurface with constant affine total curvature is an ellipsoid.

The theorem above is an analogue of the famous Hilbert's Theorem: characterizing the standard Euclidean sphere by its curvature. An affine analogue of the Minkowski Uniqueness Theorem is also obtained.

\section{INTRODUCTION}

Some basic definitions and results in the theory of affine hypersurfaces are summarized in $\S 1$. Details can be found in [C-YS], [F] and [YC].

Received by the editors November 4, 1987 and, in revised form, May 31, 1988. This paper is part of a dissertation submitted in partial fulfillment of the requirements for the degree of Doctor of Philosophy at the University of California, Los Angeles, 1986. It was presented at the American Mathematical Society 838th meeting on November 15, 1987.

1980 Mathematics Subject Classification (1985 Revision). Primary 53A15, 53A07, 52A20.

Key words and phrases. Convex affine hypersurface, Chern's conjecture, affine conormal, affine total curvature, ellipsoid. 
Let $X$ be a convex hypersurface in an $n+1$ dimensional oriented real affine space equipped with a fixed volume element. Identify the ambient affine space with $\mathbf{R}^{n+1}$ by choosing a unimodular coordinate system. An affine frame field $\left\{e_{1}, \ldots, e_{n}, b\right\}$ near a point $x$ on $X$ is given by a local tangential frame field $\left\{e_{1}, \ldots, e_{n}\right\}$, with one further local vector field $b$, such that

$$
e_{1} \wedge \cdots \wedge e_{n} \wedge b=1 \text {. }
$$

Let $e$ be the matrix $\left(e_{1}, \ldots, e_{n}\right)^{t}$, and $\phi=\left(\phi_{1}, \ldots, \phi_{n}\right)$ be the matrix of the dual frame. Then

$$
\mathrm{d} x=\phi e
$$

where " $\mathrm{d}$ " stands for exterior differentiation.

We also define the matrices of one forms $\omega, \lambda, \mu, \tau$ by

$$
\mathrm{d}\left(\begin{array}{l}
e \\
b
\end{array}\right)=\left(\begin{array}{ll}
\omega & \lambda \\
\mu & \tau
\end{array}\right)\left(\begin{array}{l}
e \\
b
\end{array}\right) \text {. }
$$

Exterior differentiating equation (1.1) gives

$$
(\operatorname{tr} \omega)+\tau=0 .
$$

Equations (1.2)-(1.4) are called the structural equations of the affine convex hypersurface $X$.

Using the fact that $\mathrm{d}^{2} x=0$ and equation (1.3), we have $\phi \lambda=0$. By Cartan's Lemma, there exists a symmetric matrix $h=\left(h_{i j}\right)$ such that $\lambda=h \phi^{t}$. As $X$ is convex, $h$ is positive definite, and the affine metric $g$ on $X$ is given by

$$
g=c^{-1} \sum_{i, j} h_{i j} \phi^{i} \phi^{j}
$$

where $c=(\operatorname{det} h)^{1 /(n+2)}$.

Let $E=\left\{E_{1}, \ldots, E_{n}\right\}$ be an orthonormal tangential frame field with respect to $g$. There exists a unique vector $B$ in $\mathbf{R}^{n+1}$ such that $E_{1} \wedge \cdots \wedge E_{n} \wedge B=1$, with the corresponding structural equations for the affine frame field $\{E, B\}$ as

$$
\begin{aligned}
\mathrm{d} x & =\Phi E, \\
\mathrm{~d}\left(\begin{array}{l}
E \\
B
\end{array}\right) & =\left(\begin{array}{ll}
\Omega & \Phi^{t} \\
M & 0
\end{array}\right)\left(\begin{array}{l}
E \\
B
\end{array}\right), \\
\operatorname{tr} \Omega & =0 .
\end{aligned}
$$

$B$ is known as the affine normal of $X$. And the affine conormal is defined to be $y=E_{1} \wedge \cdots \wedge E_{n}$. The following result is basically contained in [F]:

Proposition 1.1. Given an affine frame field $\{e, b\}$ on $X$, the affine normal $B$ is given by

$$
B=a e+c b
$$

where $c=(\operatorname{det} h)^{1 /(n+2)}$ and $a$ is determined by the equation

$$
a\left(h \phi^{t}\right)+\mathrm{d} c+c \tau=0 .
$$


It is also known that $M=-\Phi m$ for a symmetric matrix of functions $m$, which is called the third fundamental form of $X$. Note: Our definition of the third fundamental form differs from that of Cheng-Yau or Flanders by a sign.

We also define the affine mean curvature by $L=\frac{1}{n} \operatorname{tr} m$, and the affine total curvature by $K=\operatorname{det} m$.

$X$ is called an affine hypersphere if $m$ is always a multiple of the identity, i.e., $m=L I$. In this case, $X$ is called parabolic, elliptic or hyperbolic if $L$ is $=,>$ or $<0$, respectively. A theorem of Cheng-S.T. Yau [C-YS] states that elliptic or parabolic affine hyperspheres with complete Euclidean topology are hyperquadrics. Furthermore, a convex hypersurface is a non-parabolic affine hypersphere iff there exists a point $O$ in $\mathbf{R}^{n+1}$ such that $B$ is parallel to $X-O$ everywhere.

\section{AfFine CONORMAL AND THE AFFINE MEAN CURVATURE}

Let $E$ be an orthonormal basis of the affine metric of a convex hypersurface $X$ in $\mathbf{R}^{n+1}$ with affine mean curvature $L$, and $y=E_{1} \wedge \cdots \wedge E_{n}$ be the affine conormal. The following identity is known (e.g., [C-YS]):

$$
\frac{1}{n} \Delta y=-L y
$$

Proposition 2.1. Let $\left\{\nu_{1}, \ldots, \nu_{n+1}\right\}$ be the canonical basis of $\mathbf{R}^{n+1}$, and $X$ be the graph of a smooth strictly convex function: $x^{n+1}=u\left(x^{1}, \ldots, x^{n}\right)$. Then

$$
\begin{aligned}
y & \wedge \nu_{i}=-c^{-1} u_{i} \quad(1 \leq i \leq n), \\
y & \wedge \nu_{n+1}=c^{-1},
\end{aligned}
$$

where $c=\operatorname{det}\left(u_{i j}\right)^{1 /(n+2)}, u_{i}=\partial u / \partial x^{i}$ and $u_{i j}=\partial^{2} u / \partial x^{i} \partial x^{j}$.

Proof. Consider the affine frame field $\{e, b\}$ defined by $e_{i}=\left(\delta_{i 1}, \ldots, \delta_{i n}, u_{i}\right)$ and $b=(0, \ldots, 0,1)$. The dual frame is $\left\{d x^{i}\right\}$. By Flanders [F], the affine metric is $g_{i j}=c^{-1} u_{i j}$, and $c=\operatorname{det}\left(u_{i j}\right)^{1 /(n+2)}$. The volume element is $\sqrt{g} \mathrm{~d} x^{1} \wedge$ $\cdots \wedge \mathrm{d} x^{n}$ where $g=\operatorname{det}\left(g_{i j}\right)=c^{2}$.

On the other hand, let $\Phi$ be an orthonormal dual frame, then the volume element is $\Phi^{1} \wedge \cdots \wedge \Phi^{n}=c \mathrm{~d} x^{1} \wedge \cdots \wedge \mathrm{d} x^{n}$. The dual of this equation is

$$
y=E_{1} \wedge \cdots \wedge E_{n}=c^{-1} e_{1} \wedge \cdots \wedge e_{n} .
$$

Simple computations of determinants give $e_{1} \wedge \cdots \wedge e_{n} \wedge \nu_{i}=-u_{i}$ and $e_{1} \wedge$ $\cdots \wedge e_{n} \wedge \nu_{n+1}=1$. Q.E.D.

Corollary 2.2. (Affine Minkowski Uniqueness) If two affine convex hypersurfaces have the same affine conormal, then they differ by a translation.

A convex hypersurface is called affine maximal if its affine mean curvature $L$ is identically zero. We will prove a weak version of the following . 
Chern's Conjecture. Any convex graph over affine space which is an affine maximal hypersurface must be a paraboloid.

Lemma 2.3. A convex graph $x^{n+1}=u\left(x^{1}, \ldots, x^{n}\right)$ is affine maximal iff

$$
\sum_{i, j} u^{i j} \frac{\partial^{2}}{\partial x^{i} \partial x^{j}} c^{-(n+1)}=0
$$

where $\left(u^{i j}\right)=\left(u_{i j}\right)^{-1}$, and $c=\operatorname{det}\left(u_{i j}\right)^{1 /(n+2)}$.

Proof. By equations (2.1) and (2.3), we have $\frac{1}{n} \Delta c^{-1}=-L c^{-1}$. Therefore, the convex graph is affine maximal iff $\Delta c^{-1}=0$.

Instead of the Euclidean coordinates $\left(x^{i}\right)$, we will use the Legendre variables $p_{i}=u_{i}$ as the parameter. Hence, $u_{i j}=\left(\partial / \partial x^{j}\right) p_{i}$ and $u^{i j}=\left(\partial / \partial p_{j}\right) x_{i}$. Define $\tilde{g}_{i j}$ to be $g\left(\left(\partial / \partial p_{i}\right),\left(\partial / \partial p_{j}\right)\right)$. We observe that $\partial / \partial p_{i}=\sum_{k} u^{i k}\left(\partial / \partial x^{k}\right)$. As $g_{i j}=g\left(\left(\partial / \partial x^{i}\right),\left(\partial / \partial x^{j}\right)\right)=c^{-1} u_{i j}$, we have $\tilde{g}_{i j}=c^{-1} u^{i j}$. Hence, $\tilde{g}=$ $\operatorname{det}\left(\tilde{g}_{i j}\right)=c^{-2(n+1)}$.

$$
\begin{aligned}
\Delta c^{-1} & =\frac{1}{\sqrt{\tilde{g}}} \sum_{i, j} \frac{\partial}{\partial p_{i}}\left(\sqrt{\tilde{g}} \tilde{g}^{i j} \frac{\partial}{\partial p_{j}} c^{-1}\right) \\
& =c^{n+1} \sum_{i, j} \frac{\partial}{\partial p_{i}}\left(c^{-(n+1)} \cdot c u_{i j} \frac{\partial}{\partial p_{j}} c^{-1}\right) \\
& =c^{n+1} \sum_{i} \frac{\partial}{\partial p_{i}}\left(c^{-n} \frac{\partial}{\partial x^{i}} c^{-1}\right) \\
& =\frac{1}{n+1} c^{n+1} \sum_{i} \frac{\partial}{\partial p_{i}} \frac{\partial}{\partial x^{i}} c^{-(n+1)} \\
& =\frac{1}{n+1} c^{n+1} \sum_{i, j} u^{i j} \frac{\partial^{2}}{\partial x^{i} \partial x^{j}} c^{-(n+1)} \text {. Q.E.D. }
\end{aligned}
$$

We now prove the following version of Chern's Conjecture.

Proposition 2.4. Let $u$ be a smooth convex function defined on $\mathbf{R}^{n}$ with uniformly elliptic Hessian $\left(u_{i j}\right)$. If its graph is an affine maximal convex hypersurface, then $u$ is a quadratic polynomial.

Proof. Equation (2.5) tells us that the positive quantity $c^{-(n+1)}$ satisfies a second order uniformly elliptic homogeneous linear partial differential equation. By a standard result of Moser [M, p. 590], we know that $c$ must be a constant, i.e., $\operatorname{det}\left(u_{i j}\right)$ is constant. A result of Calabi [C2] then tells us that the graph of $u$ is a parabolic affine hypersphere. It thus follows from a theorem of Cheng-S.T.Yau [C-YS] that $u$ is a quadratic polynomial. Q.E.D. 


\section{AfFine CONORMAL AND THE AFFINE tOtAL CURVATURE}

Lemma 3.1. Fix an affine orthonormal frame field $E$ on $X$, with $B$ being the affine normal, and $y=E_{1} \wedge \cdots \wedge E_{n}$ the affine conormal. Then

$$
\mathrm{d} y=\sum_{i} F_{i} \Phi^{i}
$$

where

$$
F_{i}=E_{1} \wedge \cdots \wedge E_{i-1} \wedge B \wedge E_{i+1} \wedge \cdots \wedge E_{n}
$$

and

$$
\mathrm{d} F_{i}=-\sum_{j} F_{j} \Omega_{j}^{i}+M^{i} y .
$$

The proof of the lemma above is a simple computation using equations (1.6)(1.8), details can be found in [YC]. Equation (3.2) tells us that $\{F, y\}$ is a basis of $\Lambda^{n} \mathbf{R}^{n+1}$, and Lemma 3.1 implies the following

Corollary 3.2. The mapping $Y: x \mapsto y(x)$ for $x \in X$ is an immersion and defines a hypersurface in $\Lambda^{n} \mathbf{R}^{n+1}$. Moreover, $\left\{F_{i}\right\}$ forms a tangential frame field on $Y$, whose dual is given by $\Phi$.

We put

$$
\begin{aligned}
& \hat{e}=-F, \\
& \hat{b}=-y, \\
& \hat{\phi}=-\Phi .
\end{aligned}
$$

By choosing the orientation on $\bigwedge^{n} \mathbf{R}^{n+1}$ carefully, it is shown in [YC] that $\left\{\hat{e}_{1}, \ldots, \hat{e}_{n}, \hat{b}\right\}$ has unit volume. $\left\{\hat{e}_{1}, \ldots, \hat{e}_{n}, \hat{b}\right\}$ thus defines an affine frame field on $Y$. By Lemma 3.1, we have the

Corollary 3.3. The structural equations for the frame $\left\{\hat{e}_{1}, \ldots, \hat{e}_{n}, \hat{b}\right\}$ of $Y$ is

$$
\begin{aligned}
\mathrm{d} y & =\hat{\phi} \hat{e}, \\
\mathrm{~d}\left(\begin{array}{l}
\hat{e} \\
\hat{b}
\end{array}\right) & =\left(\begin{array}{cc}
-\Omega^{t} & M^{t} \\
\Phi & 0
\end{array}\right)\left(\begin{array}{l}
\hat{e} \\
\hat{b}
\end{array}\right), \\
\operatorname{tr} \Omega & =0 .
\end{aligned}
$$

From now on, we would denote the objects on $Y$ corresponding to that of $X$ in $\S 1$ by assigning hats on the tops of the respective symbols. Thus, for example, as what we did, we denote the tangent frame field by $\hat{e}$, and so forth. We hence found that

$$
\left(\begin{array}{cc}
\hat{\omega} & \hat{\lambda} \\
\hat{\mu} & \hat{\tau}
\end{array}\right)=\left(\begin{array}{cc}
-\Omega^{t} & M^{t} \\
\Phi & 0
\end{array}\right)
$$

Thus $\hat{\lambda}=M^{t}=m \hat{\phi}^{t}$ and $\hat{h}=m$. This shows the 
Lemma 3.4. If the third fundamental form $m$ of an affine hypersurface is positive definite, then its affine conormal defines another convex hypersurface in $\wedge^{n} \mathbf{R}^{n+1}$.

Proposition 3.5. If the third fundamental form of $X$ is positive definite, then $Y$ is an elliptic affine hypersphere iff the affine total curvature of $X$ is constant.

Proof. As $\hat{h}=m, \hat{c}^{n+2}=\operatorname{det} \hat{h}=\operatorname{det} m=K$, where $K$ is the total curvature. And equation (1.10) becomes

$$
\hat{a}\left(\hat{h} \hat{\phi}^{t}\right)+\mathrm{d} \hat{c}=-\hat{\tau}=0 .
$$

Thus $\hat{a}=0$ iff $\mathrm{d} \hat{c}=0$, i.e., $K$ is constant. However, by equation (1.9), the affine normal of $Y, \hat{B}=\hat{a} \hat{e}+\hat{c} \hat{b}$, is parallel to $\hat{b}=-y$ iff $\hat{a}=0$. Q.E.D.

Lemma 3.6. The affine third fundamental form of a closed convex hypersurface with constant affine total curvature is positive everywhere.

Proof. Choose a convex point $y_{0}$ on the closed immersed hypersurface $Y$. All eigenvalues of $\hat{h}=m$ will be positive at such a point. If their product is constant, all the eigenvalues of $m$ are positive everywhere, by continuity. Q.E.D.

Theorem 3.7. A closed convex affine hypersurface with constant affine total curvature is an ellipsoid.

Proof. By the previous proposition and lemma, we find that the affine conormal $Y$ of $X$ is an elliptic affine hypersphere. By a theorem of Cheng-S. T. Yau [C-YS], $Y$ is an ellipsoid. $X$ is thus also an ellipsoid by the Affine Minkowski Uniqueness Theorem 2.2. Q.E.D.

\section{ACKNOWLEDGMENT}

The author would like to thank his advisor, Professor S.-Y. Cheng for his teaching and guidance. His joint paper with Professor S.-T. Yau [C-YS] is very influential to this work. The author also thanks the referee for pointing out Lemma 3.6. It removes the superfluous assumption on positive definiteness of the affine third fundamental form when the Theorem was first formulated. Thanks are also due to Profs. Robert Greene and Barrett O'Neill for their continuous support and encouragement.

\section{REFERENCES}

[B.] W. Blaschke, Vorlesungen über Differentialgeometrie, Vol. II, Julius Springer, Berlin, 1923.

[C1.] E. Calabi, Improper affine hyperspheres of convex type etc., Michigan Math. J., 5 (1958) 105126.

[C2.] __, Complete affine hyperspheres I, Istituto Nazionale Di Alta Matematica, Symposia Mathematica, X, (1972) 19-38.

[C-YS.] S.-Y. Cheng and S.-T. Yau, Complete affine hypersurfaces, Part I, The completeness of Affine Metrics, Comm. on Pure and Applied Math., 39, (1986) 839-866.

[F.] H. Flanders, Local theory of affine hypersurfaces, J. d'Analyse Math., 15, (1965) 353-387.

[M.] J. Moser, On Harnack's Theorem for elliptic differential equations, Comm. on Pure and Applied Math., 14, (1961) 577-591.

[YC.] C.-M. Yau, Codimension two euclidean submanifolds and remarks to affine differential geometry, Ph. D. Thesis, University of California, Los Angeles, 1986.

Department of Mathematics, University of Toledo, Toledo, Ohio 43606 